\title{
X-ray structures of isopentenyl phosphate kinase
}

\author{
Mark F. Mabanglo ${ }^{\dagger}$, Heidi L. Schubert ${ }^{\ddagger}$, Mo Chen ${ }^{\dagger} \S$, Christopher P. Hill $\ddagger$, and C. Dale \\ Poultert, ${ }^{*}$ \\ †Department of Chemistry, University of Utah, 315 South 1400 East, Salt Lake City, UT 84112, USA. \\ ‡Department of Biochemistry, University of Utah School of Medicine, 15 North Medical Drive, Salt \\ Lake City, UT 84112, USA.
}

\begin{abstract}
Isoprenoid compounds are ubiquitous in nature, participating in important biological phenomena such as signal transduction, aerobic cellular respiration, photosynthesis, insect communication, and many others. They are derived from the 5-carbon isoprenoid substrates isopentenyl diphosphate (IPP) and its isomer dimethylallyl diphosphate (DMAPP). In Archaea and Eukarya, these building blocks are synthesized via the mevalonate pathway. However, the genes required to convert mevalonate phosphate (MP) to IPP are missing in several species of Archaea. An enzyme with isopentenyl phosphate kinase (IPK) activity was recently discovered in Methanocaldococcus jannaschii (MJ), suggesting a departure from the classical sequence of converting MP to IPP. We have determined the high-resolution crystal structures of isopentenyl phosphate kinases in complex with both substrates and products from Thermoplasma acidophilum (THA), as well as the IPK from Methanothermobacter thermautotrophicus (MTH), by means of single-wavelength anomalous diffraction (SAD) and molecular replacement. A histidine residue (His50) in THA IPK makes a hydrogen bond with the terminal phosphates of IP and IPP, poising these molecules for phosphoryl transfer through an in-line geometry. Moreover, a lysine residue (Lys14) makes hydrogen bonds with non-bridging oxygen atoms at $\mathrm{P}_{\alpha}$ and $\mathrm{P}_{\gamma}$ and with the $\mathrm{P}_{\beta^{-}} \mathrm{P}_{\gamma}$ bridging oxygen atom in ATP. These interactions suggest a transition state-stabilizing role for this residue. Lys14 is a part of a newly discovered "lysine triangle" catalytic motif in IPK's that also includes Lys5 and Lys205. Moreover, His50, Lys5, Lys14, and Lys205 are conserved in all IPK's and can therefore serve as fingerprints for identifying new homologues.
\end{abstract}

\section{INTRODUCTION}

Isoprenoid compounds constitute the largest and most structurally diverse family of natural products (1). The isoprenoid pathway is found in all organisms, where the molecules perform numerous functions, including roles as hormones (gibberellins, sterols), structural components in cell membranes (cholesterol, hopanoids), photosynthetic pigments (chlorophyll), visual pigments (retinal), insect pheromones (cis-verbenol), defensive agents (phytoalexins), electron carriers (ubiquinone), and protein modifiers for membrane targeting (farnesyl and geranylgeranyl diphosphate) (2-4). Many isoprenoid compounds are currently employed or

"Corresponding author: poulter@ chemistry.utah.edu.

Present address: Department of Pharmacology, University of Pennsylvania School of Medicine, 135 John Morgan Building, 3620 Hamilton Walk, Philadelphia, PA 19104-6084, USA.

Accession Codes. The coordinates of the THA IPK•IP•ATP, THA IPK•IPP•ADP and MTH IPK•GOL•ADP complex structures have been deposited in the Protein Data Bank and are listed as 3LKK, 3LL5 and 3LL9, respectively.

Supporting Information Available: Protocols for gene amplification and cloning, protein expression and purification, and metal ion dependence studies are described and Figures S1-S3 are shown. This material is available free of charge via the Internet at http://pubs.acs.org. 
under investigation as antibacterial, antifungal and anticancer drugs. The carbon skeletons of isoprenoid molecules are constructed from two 5-carbon building blocks, isopentenyl diphosphate (IPP) and its isomer dimethylallyl diphosphate (DMAPP). Addition of IPP to DMAPP and to a succession of allylic diphosphates of increasing chain length produces geranyl diphosphate $\left(\mathrm{C}_{10}\right)$, farnesyl diphosphate $\left(\mathrm{C}_{15}\right)$, geranylgeranyl diphosphate $\left(\mathrm{C}_{20}\right)$, and longer polyprenyl diphosphates $(5,6)$. These compounds lie at branch points in the isoprenoid pathway that lead to the huge variety of isoprenoid compounds found in nature $(2,7)$.

Two orthogonal routes are known for the biosynthesis of IPP and DMAPP. Acetyl CoA is the source of the carbon atoms in isoprene units synthesized in the mevalonate (MVA) pathway, while glyceraldehyde phosphate and pyruvate are used in the methylerythritol phosphate (MEP) pathway. Animals, fungi and Archaea utilize the MVA pathway exclusively. Plants utilize both pathways in distinct cellular compartments, the MVA pathway in the cytoplasm and the MEP pathway in chloroplasts. Eubacteria employ either the MVA or MEP pathway, and in a few cases, both (8).

The MVA pathway and the enzymes that catalyze the steps in the pathway are shown in Figure 1 (9). Four of the corresponding enzymes-acetoacetyl CoA thiolase, HMG CoA synthase, HMG CoA reductase, and mevalonate kinase have been identified in Archaea (10). Furthermore, Archaea have a form of IPP isomerase, the type 2 enzyme, that is genetically unrelated to the type 1 IPP isomerase found in eukaryotes (10). Homologues for the enzymes that catalyze the last two steps in the MVA pathway, phosphomevalonate kinase (PMK) and diphosphomevalonate decarboxylase (PPMD), are generally missing in Archaea. There is evidence for homologues of PMK and PPMD in species of Sulfolobus while homologues for PPMD, but not PMK, have been reported in Halobacteriales and Thermoplasmatales (10). However, these PPMD homologues do not align with Sulfolobus PPMD but rather cluster with bacterial PPMD's. Smit and Mushiegan have proposed candidate PMK and PPMD genes in Archaea (11), although these assignments have not been tested experimentally.

Recently, Grochowski et al. reported that the translated protein from MJ0044 in Methanocaldococcus jannaschii (MJ) catalyzes the ATP-dependent phosphorylation of isopentenyl phosphate (IP) rather than mevalonate or mevalonate phosphate, and the protein was assigned as an IP kinase (IPK) (12). This M. jannaschii protein and homologous proteins from Thermoplasma acidophilum (THA) and Methanothermobacter thermautotrophicus (MTH) have high catalytic efficiencies for phosphorylation of IP $\left(\mathrm{k}_{\mathrm{cat}} / \mathrm{K}_{\mathrm{m}} \sim 10^{6} \mathrm{M}^{-1} \mathrm{~s}^{-1}\right)$ (13) that are consistent with those of established enzymes in the isoprenoid pathway. Moreover, MJ0044 clusters with other genes involved in the MVA pathway and homologues of MJ0044 are found in the genomes of representatives from all of the families in Archaea (13).

Grochowski et al. suggested a variation of the "classic" MVA pathway in Archaea where mevalonate phosphate is decarboxylated to give IP, which is then converted to IPP by IPK (12).

In this paper, we present crystal structures of IPK's from THA and MTH. Structures were obtained for THA IPK in ternary complexes with the substrates, IP and ATP, and with the products, IPP and ADP. These structures reveal active site residues that participate in the phosphoryl transfer reaction.

\section{RESULTS AND DISCUSSION}

We have determined structures of IP kinases from two archaeal species, THA and MTH, with crystals obtained under a variety of conditions. All crystals were grown in the presence of IP and ATP. The THA IPK structure was determined by single wavelength anomalous diffraction (SAD) using crystals of selenomethionine-substituted protein grown in $0.1 \mathrm{M}$ citrate buffer, 
$\mathrm{pH}$ 5.0. The asymmetric unit contained two molecules of IPK, each of which contained IP and ADP in the active site. This combination of ligands presumably resulted from hydrolysis of ATP that occurred during sample preparation and crystallization. This refined structure was used as a search model in subsequent molecular replacement solutions of other IPK structures. THA IPK crystals grown in $0.1 \mathrm{M}$ malonate/imidazole/borate (MIB) buffer, $\mathrm{pH} 7.0$, also contained two molecules of the enzyme in the asymmetric unit but each contained the substrates IP and ATP in the active site (IPK•IP•ATP). In addition, THA IPK crystals grown in propionate/cacodylate/Bis-Tris (PCB) buffer, $\mathrm{pH} 6.0$ contained four molecules in the asymmetric unit where one molecule contained IP and ATP while the other three contained the products, IPP and ADP, formed during crystallization. The presence of substrate and product complexes in the asymmetric unit is reasonable given an equilibrium constant for the reaction, $\mathrm{K}_{\mathrm{eq}}=6.3$, for uncomplexed substrates and products (13). Finally, a structure for MTH IPK in complex with glycerol and ADP (IPK•GOL•ADP) was determined. All of these structures were refined to $\mathrm{R}_{\text {free }}$ values of $23-26 \%$ against data to $2.0-2.15 \AA$ resolution. All four structures have excellent geometry, with more than $95 \%$ of the residues in favored Ramachandran regions. Crystallographic statistics are given in Table 1.

All of the crystallized proteins of THA IPK included N-terminal Phe $(-1)$ and $\operatorname{Thr}(0)$ residues that were the remnants of a TEV protease recognition site. In some cases, one or both of these residues were visible in the electron density. Moreover, electron density for residues Asp $(-3)$ and Pro(-2), also parts of the TEV protease recognition site were found in the asymmetric unit of the crystal grown in PCB buffer. This asymmetric unit was interesting because of the combination of substrates and products in the molecules mentioned earlier, as well as the significant movement of loops in two molecules. Briefly, molecules A, B and C contained bound products, while molecule D contained bound substrates. Molecules B and D formed the only homodimer in the asymmetric unit, resulting in an interesting mix of ternary enzyme complexes in one dimeric structure. In molecules $\mathrm{C}$ and $\mathrm{D}$, the $\eta 1-\alpha \mathrm{A}$ loop was in a conformation different than those in molecules $\mathrm{A}$ and $\mathrm{B}$, which allowed these molecules to make crystal lattice contacts through Tyr17 (Supplementary Figure 1, panel a). This loop movement resulted in a significant shift in the side chain position of Lys14, an invariant residue in IPK's with direct involvement in catalysis that will be discussed later. Moreover, in molecules $\mathrm{C}$ and $\mathrm{D}$, alternate conformations were found for another catalytic residue, His50 (Supplementary Figure 1, panel b). An active site water molecule found in enzymes structurally related to IPK was found only in molecules A, C and D. The unique characteristics of these asymmetric unit molecules allowed us to choose molecule $\mathrm{A}$ as the best structure that represents the THA IPK product complex (IPK・IPP•ADP).

The two asymmetric unit molecules of IPK・IP•ATP were almost identical and both contained IP and ATP in the active site. The same is true for the MTH structure, except that the asymmetric unit molecules contained ADP and either glycerol or water molecules in the IP binding site. This glycerol molecule presumably displaced a bound IP molecule when the crystal was being prepared for cryogenic data collection.

\section{Fold and quaternary structure of IPK}

IPK belongs to the amino acid kinase (AAK) family of enzymes that also includes $\mathrm{N}$-acetylL-glutamate kinase (NAGK) (14), the aspartokinase (AK) N-terminal domain (15), glutamate-5-kinase (G5K) (16), carbamate kinase (CK)(17), uridine monophosphate kinase (UMPK) (18) and fosfomycin kinase (FomA) (19). This family is characterized by the open $\alpha \beta \alpha$ sandwich fold first observed in E. faecalis CK (17) and catalyzes the transfer of a phosphoryl group from a nucleotide to a small molecule acceptor. Depending on the functional group that is phosphorylated, this family can be divided into two subdivisions. Enzymes in the "carboxylate" subdivision catalyze the transfer of a phosphoryl group to a carboxylate or 
carbamate group (NAGK, CK, G5K and AK), while those in the "phosphate" subdivision transfer a phosphoryl group to a phosphate or phosphonate (UMPK, FomA, IPK). THA IPK is structurally most similar to FomA, where the backbone RMSD is $2.6 \AA$ on 224 residues. Overlap of FomA and NAGK with THA IPK also aligns their respective substrates and most of their active site residues, suggesting similar catalytic mechanisms. As expected, MTH IPK shares high structural homology with THA IPK with RMSD of $1.9 \AA$ Aver $223 \mathrm{C} \alpha$ atoms, although sequence identity is only $32 \%$. These structural comparisons confirm the correct assignment of THA and MTH IPK's to the AAK family of enzymes.

The THA IPK structure contains an N-terminal domain (residues 1-155) that binds IP and a C-terminal domain (residues 156-245) that binds ATP. Each molecule of THA IPK consists of $16 \beta$-strands, eight $\alpha$-helices and two $3_{10}$ helices interconnected by loops and turns (Figure 2 , panel a). The open $\alpha \beta \alpha$ sandwich fold formed by these secondary structures is highly similar to that of NAGK (14): the same structural elements form the central $\beta$-sheet core $(\beta 5, \beta 8, \beta 2$, $\beta 1, \beta 11, \beta 15, \beta 16, \beta 14)$ and the two flanking layers of $\alpha$-helices $(\alpha \mathrm{C}, \alpha \mathrm{A}, \alpha \mathrm{H}$ on one side and $\alpha \mathrm{D}, \alpha \mathrm{E}, \alpha \mathrm{G}, \alpha \mathrm{F}$ on the other). Three $\beta$-hairpin turns reside in the $\mathrm{N}$-terminal domain, two of which form the surrounding walls of the aliphatic tail of IP $(\beta 3-\beta 4, \beta 9-\beta 10)$ and another forming one side of the IP binding pocket ( $\beta 6-\beta 7$ ). The $\beta 3-\beta 4$ hairpin turn of THA IPK is shorter than that of NAGK but is in the same closed conformation that allows it to cap the IP binding site and shield its hydrophobic surroundings from solvent molecules (14). The remaining $\beta 12-\beta 13$ hairpin turn resides in the $\mathrm{C}$-terminal domain and hovers above the purine ring of ATP. This $\beta$-hairpin is found within the segment that connects the core $\beta$-sheet strands $\beta 11$ and $\beta 14$ and is longer than the corresponding segment in NAGK. The longest helix in THA IPK, $\alpha \mathrm{C}$, aligns well with $\alpha \mathrm{C}$ in NAGK, but is one turn longer.

The segment between $\beta 14$ and $\alpha \mathrm{G}$ (residues 189-201) in the THA IPK structure is disordered in all molecules in the asymmetric unit, as evidenced by an un-interpretable electron density. This segment contains the helix, $\alpha \mathrm{F}$ and the $\alpha \mathrm{F}-\alpha \mathrm{G}$ junction loop that form one side of the ATP binding site. The corresponding segment in MTH IPK is missing (204-218), as is also seen in one molecule of G5K (2J5T) (16), suggesting that the flexibility of these secondary structures might be important for the binding and release of ATP.

The THA and MTH IPK's are dimeric in the crystal, consistent with the observed oligomerization state during gel filtration chromatography (Figure 3, panels and $b$ ). The dyadic axis is perpendicular to the core $\beta$-sheet that runs across the dimer (16 $\beta$-sheets, 8 per subunit). As in all enzymes of this family that catalyze phosphoryl transfer, each active center is confined to a single subunit. However, dimer formation may be structurally significant since $\mathrm{N}$-terminal domain loops surrounding the active site are anchored to the other subunit of the dimer (14).

Analysis of the dimerization surface of THA IPK using the EBI PISA (20) server resulted in a complexation significance score of 0.797 , corresponding to a tight dimer that buries a total of $3275 \AA^{2}$ of solvent accessible area between the two monomers. The compactness arises from a total of nine hydrogen bonding interactions between residues in the long interfacial $\alpha \mathrm{C}$ helices of both subunits and between the $\alpha \mathrm{D}$ helix of one subunit and the $\beta 4, \beta 6$, and $\beta 9$ strands and the $\eta 2$ helix of the other. Eight electrostatic interactions between the $\alpha \mathrm{C}$ helices of the two subunits and between the $\beta 6$ strand of one subunit and the $\beta 7$ strand of the other further stabilize the dimer. Finally, hydrophobic interactions mediated by residues in the $\alpha \mathrm{C}, \alpha \mathrm{D}$ and $\eta 3$ helices, and the $\beta 4, \beta 5, \beta 6, \beta 9$ and $\beta 10$ strands further bind the two molecules.

Although essentially the same secondary structures are used by the AAK enzymes in dimerization, different quaternary architectures are generated by changing the angle between the long interfacial helices $\alpha \mathrm{C}$ of the dimer subunits (16). In the case of THA IPK, the crossover point of these helices is found on the fifth turn from the $\mathrm{N}$-terminus, and the rotation angle 
around an axis that penetrates the inter-subunit interface perpendicularly at this point is $\sim 220^{\circ}$. In MTH IPK, the crossover point shifts to the sixth turn from the N-terminus while the rotation angle remains at $\sim 220^{\circ}$. In NAGK and CK the crossover points in $\alpha \mathrm{C}$ occur in the third turn, and shifts to the fifth turn in G5K, while the rotation angles are $110^{\circ}$ in both NAGK and CK and $260^{\circ}$ in G5K (16).

\section{The active site}

The THA IPK structures presented here are the first to show the molecular details of binding IP and IPP molecules by an enzyme (Figure 4, panels a and b). Many characteristics of AAK enzymes have been described previously in the discussion of the NAGK structure, and several of these traits are conserved in both THA and MTH IPK's. Briefly, the IP (IPP) binding pocket is located in the $\mathrm{N}$-terminal domain formed by the $\beta 2-\alpha \mathrm{B}$ loop, the $\alpha \mathrm{B}$ helix, the $\beta 3-\beta 4$ hairpin, the N-terminus of the $\alpha \mathrm{C}$ helix, the $\beta 8-\beta 9$ loop, the $\beta 9-\beta 10$ hairpin turn, and the $\beta 10-\alpha \mathrm{E}$ loop. The $\alpha \mathrm{B}$ helix contributes to binding IP (IPP) through the orientation of the positive end of the helix dipole and the hydrogen bonding and electrostatic interactions made by the charged His50 residue. In the FomA structure, the $\alpha \mathrm{B}$ helix is ordered in the presence of fosfomycin and disordered in its absence (19). Comparison of AAK enzyme structures revealed that only the enzymes in the "phosphate" subdivision (UMPK, FomA and IPK) align their respective $\alpha B$ helices. Moreover, an aligning residue at the His50 position is found exclusively in these enzymes.

The His50 residue in IPK•IP•ATP poise the bound IP for nucleophilic attack on $\mathrm{P}_{\gamma}$ of ATP by hydrogen bonding with a non-bridging oxygen atom in the terminal phosphate of IP. In the IPK・IPP•ADP structure, this residue moves slightly to make a hydrogen bond with a nonbridging oxygen atom in the IPP terminal phosphate (Figure 4, panel b), facilitating the reverse reaction. In fact, in comparing IPK•IP•ATP and IPK•IPP•ADP (r.m.s.d. $0.4 \AA$ ), the slight change in the position of His50 to hydrogen bond with the terminal phosphate of the product is one of the very few coordinate shifts that were observed. In FomA, His58 positions fosfomycin for nucleophilic attack on the phosphate donor through hydrogen bonding with an intervening water molecule (19). The corresponding residue in E. coli UMPK, Arg62, hydrogen bonds with the terminal phosphates of both substrate and product and stabilize $\mathrm{P}_{\gamma}$ of ATP through electrostatic interactions (21). These observations together with the invariance of this histidine residue in putative IPK homologues (13,Supporting Information, Table S2), the apparent pKa of 6.5 for THA IPK ( 6.8 for MTH IPK) from $\mathrm{pH}$ dependence studies (13) suggest that this His50 may perform direct catalytic roles similar to those proposed for Arg62 in UMPK, besides being a signature trait of IPK's.

The specificity of IPK arises from hydrophobic residues that cradle the aliphatic portion of IP and a constellation of conserved residues that hydrogen bond with its phosphate moiety (Figure 4 , panel a). The residues Met77 and Val73 ( $\alpha \mathrm{C}$ N-terminus), Val130 and Ile140 ( $\beta 9-\beta 10$ hairpin) and Ala53 ( $\alpha \mathrm{B}$ C-terminus) and Tyr127 ( $\beta 8-\beta 9$ loop) all form the hydrophobic pocket for the apolar tail of IP. In addition, Leu58 and Pro59 of the $\beta 3-\beta 4$ hairpin enhance the hydrophobic surroundings by capping the IP binding pocket. Notably, the $\beta 3-\beta 4$ hairpin is found only in the structures of NAGK and IPK. This motif is in the closed conformation in substrate-bound NAGK structures (14) as in the IPK•IP•ATP and IPK•IPP•ADP structures. The side chains of some, if not all, of these hydrophobic residues may give rise to the chain length specificity of IPK. Geranyl phosphate, which is one isoprene unit longer than IP, is a poor substrate for IPK, indicating that the IP binding site can not easily accommodate a $\mathrm{C}_{10}$ isoprenoid chain (13).

The phosphate moiety of IP is recognized in the active site by structural motifs that are wellconserved in the AAK family. In IPK•IP•ATP, the three non-bridging oxygen atoms of IP make hydrogen bonding interactions with the $N_{\varepsilon}$ of His 50 , the $N$ atom of Gly45 in the $\beta 2-\alpha B$ 
loop, and Asp144 through a bound water molecule (Figure 4, panel a). This water molecule is found in the holoenzyme structures of E. coli NAGK (pdb ID 1GS5), P. furiosis UMPK (pdb ID 2BMU), E. coli UMPK (pdb ID 2BNE) and FomA (pdb ID 3D41). A water molecule in a similar position is present in IPK•IPP•ADP (Figure 4 , panel b). The $\beta 2-\alpha \mathrm{B}$ loop, which contains Gly45, is one of the conserved glycine-rich loops in the AAK family and is thought to stabilize the transition state intermediate and the phosphorylated product of these enzymes $(19,22)$. In IPK•IPP•ADP, Gly45 of this loop makes a hydrogen bond with the $\mathrm{P}_{\alpha}-\mathrm{P}_{\beta}$ bridging oxygen atom of IPP. The other glycine-rich loop, $\beta 1-\eta 1$, contains Gly 8 that stabilizes IPP by making a hydrogen bond with a non-bridging oxygen atom of its terminal phosphate.

The $\beta 10-\alpha E$ loop completes the structural elements involved in binding the polar end of IP. This loop contains Ser142, which is conserved in the "phosphate" subdivision (Thr120 in $P$. furiosis UMPK, Ser149 in FomA) $(18,19)$ and makes a hydrogen bond with the terminal phosphate of the substrates. In IPK・IP•ATP and IPK・IPP•ADP, the orientation of the side chain hydroxyl group of Ser142 does not allow a hydrogen bond with substrates or products (Figure 4 , panel a and b). However, an alternate rotamer could provide hydrogen bond stabilization as the substrates moved to the transition state. The positive end of the helix dipole of $\alpha \mathrm{E}$, initiated by the $\beta 10-\alpha E$ loop, may further stabilize the polar head of the IP molecule as well as the negatively-charged transition state intermediate, as in other structures of AAK enzymes.

Thus, the specificity of IPK towards IP arises from a combination of hydrophobic interactions with the hydrocarbon tail and hydrogen bonding and electrostatic interactions with the phosphate head group to match the amphiphatic character of IP. This differentiates the IPK active site from other enzymes in the family whose substrates do not have this intrinsic amphiphathicity, such as that of NAGK whose active site is laden with both polar and charged residues that interact with the acetyl and carboxyl groups of NAG. This unique binding elements of IPK limits its catalytic promiscuity.

The location of the ATP binding site in both THA and MTH IPK's superimpose well with those of NAGK and other AAK enzymes. Conserved amino acid residues in these enzymes that have previously been identified to form hydrogen bonding, electrostatic and hydrophobic interactions with the purine ring, sugar and polyphosphate moieties of ATP are also found in IPK. For instance, the NAGK residues Met214 (purine stacking), Asp181 (ribose 3'-OH binding), Asp162 (polyphosphate positioning) and Lys8 (phosphate hydrogen bonding) (14) are also conserved in THA IPK as Ile202, Asp164, Asp144 and Lys5 respectively. In MTH IPK, the corresponding residues are Met220, Asp178, Asp158 and Lys5. Other ATP-binding residues in THA IPK that superimpose with similar ATP-binding residues in NAGK are shown in (Figure 5).

The $\beta 1-\eta 1$ glycine-rich loop that contains Gly8 engages the $\mathrm{P}_{\beta}$ and $\mathrm{P}_{\gamma}$ of ATP in IPK•IP•ATP through hydrogen bonding. These interactions by the conserved Gly 8 with the phosphate groups ATP (and with the terminal phosphate of IPP stated earlier) are seen for the corresponding residue in other enzymes and reflect a role in phosphoryl transfer $(21,22)$. The residue Asp172 and Lys174 form hydrogen bonds with the 2'- and 3'-OH groups of ATP.

Finally, a hydrogen bond with side chain carboxylate of Asp164 completes an intricate network of interactions that secure this end of ATP, thus positioning the polyphosphate end for nucleophilic attack by IP.

As stated earlier, $\alpha \mathrm{F}$ and the loop that connects it to $\alpha \mathrm{G}$ were not modeled in our IPK structures. These secondary structures contain Phe191, analogous to Trp202 in FomA, which stacks on the adenine ring, and the conserved DVTGG sequence that make important binding interactions with the nucleotide seen in other AAK enzymes. We suspect that these interactions are more or less conserved in IPK, although a more complete IPK structure would be necessary to 
unequivocally establish the nature of these interactions. The flexibility and location of these absent secondary structures suggest a role in nucleotide sequestration and release.

The IPK•IP•ATP structure was obtained without resorting to a substitution of the $\mathrm{P}_{\beta}-\mathrm{P}_{\gamma}$ bridging oxygen atom that would prevent phosphoryl transfer. In addition, phosphoryl transfer between added substrates during the crystallization experiments provided IPK•IPP•ADP. These two structures, providing snapshots of the IPK enzyme before and after catalysis, suggest an important role for Lys14 in stabilizing the transition state (Figure 6, panel a and b). This residue is also conserved in FomA and homologs of IPK (13,Supporting Information, Table S2). An associative mechanism of phosphoryl transfer was proposed for NAGK (14). The similarity of the over-all fold and the superposition of the substrate and nucleotide binding sites of THA and MTH IPK's with those of NAGK suggests a similar mechanism for IPK (Figure 7). In IPK•IP•ATP, a nucleophilic oxygen atom the phosphate group in IP is $2.9 \AA$ from the electrophilic $\mathrm{P}_{\gamma}$ phosphate atom in ATP, and is poised for attack. The products in IPK•IPP•ADP are likewise poised for the reverse reaction, where the nucleophilic oxygen atom in $\mathrm{P}_{\beta}$ of ADP is $2.3 \AA$ from the electrophilic $\mathrm{P}_{\beta}$ phosphate atom of IPP.

As in NAGK, the conserved glycine rich loops $\beta 1-\eta 1$ and $\beta 2-\alpha B$ of THA IPK, the positive helix dipoles of $\alpha \mathrm{B}$ and $\alpha \mathrm{E}$, the positive charges of Lys5 and Lys205, and Asp144, which positions Lys5 and Lys205 towards the polyphosphates, create an environment for stabilizing the negatively charged transition state (Figure 7, panels a-c). A divalent metal ion, $\mathrm{Mg}^{2+}$, further contributes to stabilization by the same mechanism in addition to its effect on the orientation of the ATP polyphosphate (14). In IPK•IP•ATP and IPK・IPP•ADP, a $\mathrm{Mg}^{2+}$ ion could not be unambiguously assigned to the electron density that superimposes onto the $\mathrm{Mg}^{2+}$ atom in the structure of NAGK. Instead, a water molecule is assigned to this density since the distance of the sphere's centroid to the relevant polyphosphate oxygen atoms are more consistent with hydrogen bonding than with metal coordination. Nevertheless, product turnover assays using radioactive $\left.{ }^{32} \mathrm{P}\right] \mathrm{ATP}$ (13) and $\mathrm{Mg}^{2+}$ confirmed the divalent metal ion dependence of IPK, a well-established characteristic of kinases (Supplementary Figure 3, panel a)(23). IPK was also active in the presence of $\mathrm{Mn}^{2+}, \mathrm{Zn}^{2+}, \mathrm{Ni}^{2+}, \mathrm{Cd}^{2+}$, and $\mathrm{Co}^{2+}$ (Supplementary Figure 3, panel b).

In IPK・IP•ATP, Lys 14 is located within hydrogen bonding distance of the non-bridging oxygen atoms of $\mathrm{P}_{\alpha}(3.0 \AA)$ and $\mathrm{P}_{\gamma}(2.8 \AA)$ and the $\mathrm{P}_{\beta}-\mathrm{P}_{\gamma}$ bridging oxygen atom (3.1 $)$ in ATP. In IPK・IPP•ADP, Lys 14 makes a hydrogen bond with a non-bridging oxygen atom of $\mathrm{P}_{\beta}(2.7 \AA)$ in IPP and the non-bridging oxygen atoms of $\mathrm{P}_{\alpha}(2.7 \AA)$ and $\mathrm{P}_{\beta}(3.0 \AA)$ of ADP. Interestingly, a corresponding residue, Lys 18, is found in the (FomA $\bullet$ fosfomycin•AMPPNP) complex (19). However, in this structure, Lys 18 only forms a hydrogen bond with a $\mathrm{P}_{\gamma}$ oxygen atom in AMPPNP, which in turn, is not properly oriented for nucleophilic attack by fosfomycin. The relative positions of IP and ATP in THA IPK are consistent with phosphoryl transfer. Thus, in THA IPK Lys5, Lys14 and Lys205 form a "lysine triangle" surrounding the phosphate residues in the substrates of IPK's.

Along with His50, Lys14 is a conserved feature of the IPK's. These residues may have evolved to preferentially stabilize transition states that contain more negative charge than those formed during phosphoryl transfers involving carboxylated and carbamated substrates, thus giving rise to IP kinases. The conservation of these traits in FomA along with other key catalytic residues in seen in IPK is also consistent with the acquisition of antibiotic resistance toward fosfomycin in Streptomyces by a lateral gene transfer of an ancestral IPK gene from Archaea.

\section{Conclusion}

The crystal structures of IPK from two species of Archaea are highly similar despite their low amino acid sequence identities and key catalytic residues are conserved in both proteins. The 
catalytic machinery of Archaeal IPK's is related to that found in other AAK enzymes except for the incorporation of two additional residues, His50 and Lys14, in the active site. The amino acids appear to orient the phosphate residues in the bound substrates and are positioned to stabilize the transition state for phosphoryl transfer. Moreover, a catalytic lysine triangle formed by Lys5, Lys14 and Lys205 constitutes a new catalytic motif in IPK's. The presence of these key IPK residues in bacterial FomA suggests that fosfomycin resistance in Streptomyces was acquired by a horizontal transfer of an Archaeal gene for IPK genes. Finally, homologues of IPK are found in the genomes of representatives from all of the families of Archaea. Although the existence of a PMD is yet to be established, it is likely that isoprenoid compounds are synthesized by the alternate MVA pathway in these organisms.

\section{METHODS \\ Crystallization}

All crystals of THA IPK were grown by the sitting drop vapor diffusion method at $21{ }^{\circ} \mathrm{C}$ using a protein mixture comprising $0.30 \mathrm{mM}$ protein, $1.5 \mathrm{mM} \mathrm{MgCl} 2,1.5 \mathrm{mM}$ IP and $1.5 \mathrm{mM}$ ATP that was prepared and incubated for at least two $h$ before crystallization. In all cases the crystals grew to maximum dimensions in about one week in drops comprising a 1:1 mixture of protein and reservoir solutions.

Selenomethionine-substituted THA IPK crystals complexed with IP and ADP were grown using a solution of $0.1 \mathrm{M}$ sodium citrate, $\mathrm{pH} 5.0$, and 15\% PEG 6000. Crystals grew to maximum dimensions in about one week. The IPK・IP•ATP crystal was grown using native protein and a reservoir solution of 0.1M MIB buffer (2:3:3 molar ratio of sodium malonate, imidazole and boric acid), pH 7.0 and 25\% PEG 1500. The IPK•IPP•ADP crystal was obtained using selenomethionine-substituted protein and a reservoir solution of $0.1 \mathrm{M} \mathrm{PCB}$ buffer $(2: 1: 2$ molar ratio of sodium propionate, sodium cacodylate and Bis-Tris propane) $\mathrm{pH} 6.0$ containing $25 \%$ PEG 1500. In this case the substrate included with the crystallized protein was converted and preferentially bound as product under the crystallization conditions.

Crystals of MTH IPK grew by mixing $2 \mu \mathrm{L}$ protein $\left(5 \mathrm{mg} \mathrm{mL}^{-1}\right.$ MTH IPK, $5 \mathrm{mM} \mathrm{MgCl} 2,1$ $\mathrm{mM}$ ATP) with a precipitant solution containing $12 \%$ PEG 6000 and $2 \mathrm{M} \mathrm{NaCl}$. The active site was found to contain glycerol and ADP.

\section{Crystal structure determination}

Prior to data collection, all crystals were cryo-protected by immersion for $30 \mathrm{~s}$ in a modified reservoir solution that contained $30 \%$ glycerol and cooled by plunging into liquid nitrogen. Diffraction data were collected at $100 \mathrm{~K}$ on a rotating anode source or at beamline 11-1 of the SSRL synchrotron and processed using HKL2000 (25). The THA IPK structure in complex with IP and ADP was determined by single-wavelength anomalous diffraction (SAD) using PHENIX (26). A total of 45 selenium sites were located and used to calculate initial phases to $1.90 \AA$, and the phase estimates were improved by density modification to generate a readily interpretable electron density map. Model building was done using COOT (27). Refinement used REFMAC5 (28) with the final refinement cycles performed using PHENIX (25). This refined model was used to determine all of the other structures by molecular replacement using PHASER (29) in the CCP4 suite (30).

The DaliLite server (31) was used to superimpose the $\mathrm{C} \alpha$ atoms of two protein structures and measure the RMSD between the two. Molprobity (32) was used for structure validation. PyMol (33) and the command "Ray" were used to create images of the protein structures. 


\section{Supplementary Material}

Refer to Web version on PubMed Central for supplementary material.

\section{Acknowledgments}

We thank J. Muller of the Department of Chemistry of the University of Utah for assistance in mass spectrometry. This research was supported by NIH grants GM 25521 (CDP) and GM 56775 (HLS, CPH). Part of the work was carried out at the Stanford Synchrotron Radiation Light Source (SSRL), a national research facility operated by Stanford University on behalf of the Department of Energy, Office of Basic Energy Sciences. The SSRL Structural Molecular Biology Program is supported by the Department of Energy, Office of Biological and Environmental Research and by the US National Institute of Health (NIH), National Center for Research Resources, Biomedical Technology Program, and the National Institute of General Medical Sciences.

\section{REFERENCES}

1. Reiling KK, Yoshikuni Y, Martin VJJ, Newman J, Bohlmann J, Keasling JD. Mono and diterpene production in Escherichia coli. Biotechnol. Bioeng 2004;87:200-212. [PubMed: 15236249]

2. Davis EM, Croteau R. Cyclization enzyme in the biosynthesis of monoterpenes, sesquiterpenes, and di-terpenes. Top. Curr. Chem 2000;209:53-95.

3. Liang P, Ko T, Wang AH. Structure, mechanism and function of prenyltransferases. Eur. J. Biochem 2002;269:3339-3354. [PubMed: 12135472]

4. Sacchetini JC, Poulter CD. Creating isoprenoid diversity. Science 1997;277:1788-1789. [PubMed: 9324768]

5. Ogura K, Koyama T. Enzymatic aspects of isoprenoid chain elongation. Chem. Rev 1998;98:12631276. [PubMed: 11848932]

6. Poulter CD. Farnesyl diphosphate synthase. A paradigm for understanding structure and function relationships in E-polyprenyl diphosphate synthases. Phytochem. Rev 2006;5:17-26.

7. Song L, Poulter CD. Yeast farnesyl diphosphate synthase: Site-directed mutagenesis of residues in highly conserved prenyltransferase domains I and II. Proc. Nat. Acad. Sci. USA 1994;91:3044-3048. [PubMed: 8159703]

8. Rohdich F, Bacher A, Eisenreich W. Perspectives in anti-infective drug design. The late steps in the biosynthesis of the universal terpenoid precursors, isopentenyl diphosphate and dimethylallyldiphosphate. Bioorg. Chem 2004;32:292-308. [PubMed: 15381396]

9. Kuzuyama T. Mevalonate and nonmevalonate pathways for the biosynthesis of isoprene units. Biosci. Biotechnol. Biochem 2002;66:1619-1627. [PubMed: 12353619]

10. Boucher Y, Kamekura M, Doolittle WF. Origins and evolution of isoprenoid biosynthesis in Archaea. Mol. Microbiol 2004;52:515-527. [PubMed: 15066037]

11. Smit A, Mushiegan A. Biosynthesis of isoprenoids via mevalonate in Archaea: the lost pathway. Genome Res 2000;101:1468-1484. [PubMed: 11042147]

12. Grochowski LL, Xu HM, White RH. Methanocaldococcus jannaschii uses a modified mevalonate pathway for biosynthesis of isopentenyl diphosphate. J. Bacteriol 2006;188:3192-3198. [PubMed: 16621811]

13. Chen M, Poulter CD. Characterization of thermophilic and archaeal isopentenyl phosphate kinases. Biochemistry 2010;49:207-217. [PubMed: 19928876]

14. Ramon-Maiques S, Marina A, Gil-Ortiz F, Fita I, Rubio V. Structure of acetylglutamate kinase, a key enzyme for arginine biosynthesis and a prototype for the amino acid kinase family, during catalysis. Structure 2002;10:329-342. [PubMed: 12005432]

15. Liu X, Pavlovsky AG, Viola RE. The structural basis for allosteric inhibition of a threonine-sensitive aspartokinase. J. Biol. Chem 2008;283:16216-16225. [PubMed: 18334478]

16. Marco-Marin C, Gil-Ortiz F, Perez-Arellano I, Cervera J, Fita I, Rubio V. A novel two-domain architecture within the amino-acid kinase enzyme family revealed by the crystal structure of Escherichia coli glutamate 5-kinase. J. Mol. Biol 2007;367:1431-1446. [PubMed: 17321544] 
17. Marinaz A, Alzari PM, Bravo J, Uriarte M, Barcelona B, Fita I, Rubio V. Carbamate kinase: new structural machinery for making carbamoyl phosphate, the common precursors of pyrimidines and arginines. Protein Science 1999;8:934-940. [PubMed: 10211841]

18. Marco-Marin C, Gil-Ortiz F, Rubio V. The crystal structure of Pyrococcus furiosis UMP kinase provides insight into catalysis and regulation in microbial pyrimidine nucleotide biosynthesis. J. Mol. Biol 2005;352:438-454. [PubMed: 16095620]

19. Pakhomova S, Bartlett S, Augustus A, Kuzuyama T, Newcomer M. Crystal structure of fosfomycin resistance kinase FomA from Streptomyces wedmorensis. J. Biol. Chem 2008;283:28518-28526. [PubMed: 18701452]

20. Krissinel E, Henrick K. Inference of macromolecular assemblies from crystalline state. J. Mol. Biol 2007;372:774-797. [PubMed: 17681537]

21. Briozzo P, Evrin C, Meyer P, Assairi L, Joly N, Barzu O, Gilles AM. Structure of Escherichia coli UMP kinase differs from that of other nucleoside monophosphate kinases and sheds new light on enzyme regulation. J. Biol. Chem 2005;280:25533-25540. [PubMed: 15857829]

22. Gil-Ortiz F, Ramon-Maiques S, Fita I, Rubio V. The course of phosphorous in the reaction of Nacetyl-L-glutamate kinase, determined from the structures of crystalline complexes, including a complex with AlF(4)(-) transition state mimic. J. Mol. Biol 2003;331:231-244. [PubMed: 12875848]

23. Tian G, Kane LS, Holmes WD, Davis ST. Modulation of cyclin-dependent kinase 4 by binding of magnesium (II) and manganese (II). Biophys Chem 2002;95:79-90. [PubMed: 11880175]

24. Studier FW. Protein production by auto-induction in high density shaking cultures. Protein Expr. Purif 2005;41:207-234. [PubMed: 15915565]

25. Otwinowski, Z.; Minor, W. Processing of X-ray diffraction data collected in oscillation mode. In: Carter, CW., Jr; Sweet, RM., editors. Methods in Enzymology, Macromolecular Crystallography. Vol. Vol. 276. New York: Academic Press; 1997. p. 307-326.

26. Adams PD, Afonine PV, Bunkóczi G, Chen VB, Davis IW, Echols N, Headd JJ, Hung L-W, Kapral GJ, Grosse-Kunstleve RW, McCoy AJ, Moriarty NW, Oeffner R, Read RJ, Richardson DC, Richardson JS, Terwilliger TC, Zwart PH. PHENIX: a comprehensive Python-based system for macromolecular structure solution. Acta Cryst.D 2010;66:213-221. [PubMed: 20124702]

27. Emsley P, Cowtan K. Coot: model-building tools for molecular graphics. Acta Crystallogr 2004;60:2126-2132.

28. Murshudov GN, Vagin AA, Dodson EJ. Refinement of macromolecular structures by the maximumlikelihood method. Acta Crystallogr.D 1997;53:240-255. [PubMed: 15299926]

29. McCoy AJ, Grosse-Kunstleve, Adams PD, Winn MD, Storoni LC, Read RJ. Phaser crystallographic software. J. Appl. Cryst 2007;40:658-674. [PubMed: 19461840]

30. Collaborative Computational Project, Number 4. The CCP4 Suite: programs for protein crystallography. Acta Crystallogr.D 50:760-763.

31. Holm L, Park J. DaliLite workbench for protein structure comparison. Bioinformatics 2000;16(6): 566-567. [PubMed: 10980157]

32. Davis IW, Leaver-Fay A, Chen VB, Block J, Kapral GJ, Wang X, Murray L, Arendall B III, Snoeyink J, Richardson JS, Richardson DC. Molprobity: all-atom contacts and structure validation for proteins and nucleic acids. Nucleic Acids Res 2007;35(Web Server issue):W375-W383. [PubMed: 17452350]

33. DeLano, WL. The PyMOL Molecular Graphics System, DeLano Scientific. CA, USA: Palo Alto; 2002. 

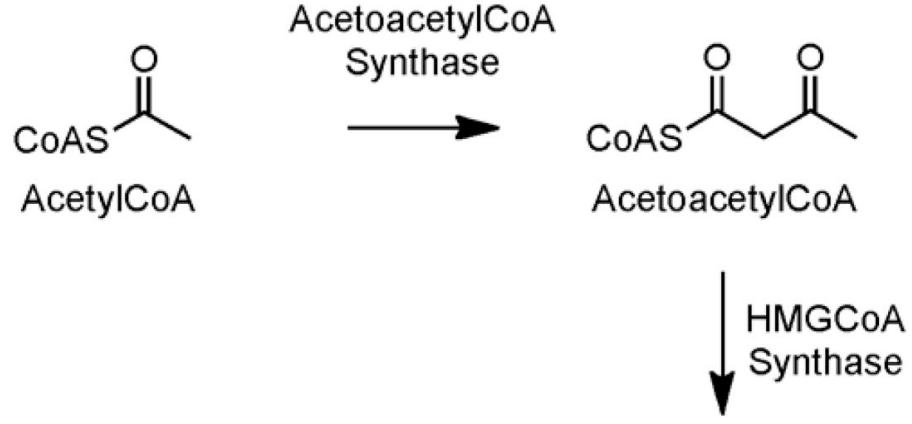<smiles>C[C@](O)(CCO)CC(=O)[O-]</smiles>

Mevalonate

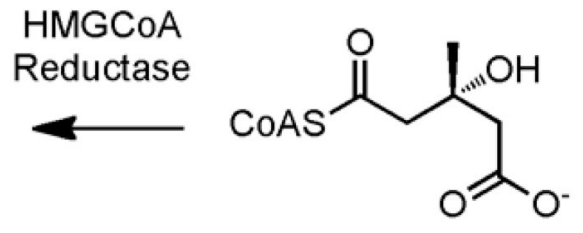

HMGCoA

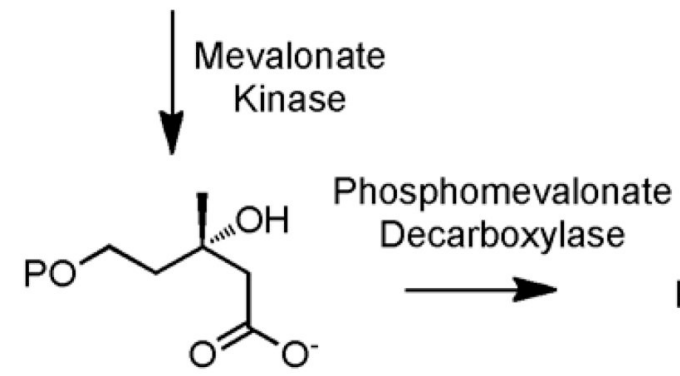

Phosphomevalonate<smiles>C=C(C)CCOP</smiles>

Isopentenyl

Phosphate
<smiles>C[C@](O)(CCOP)CC(=O)[O-]</smiles>

Diphosphomevalonate

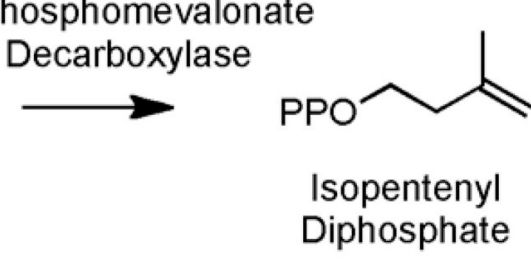

Figure 1.

The alternate route in the MVA pathway. In Archaea, the last two steps in the formation of IPP are reversed. Phosphomevalonate is decarboxylated to form IP, which is then phosphorylated to give IPP. 


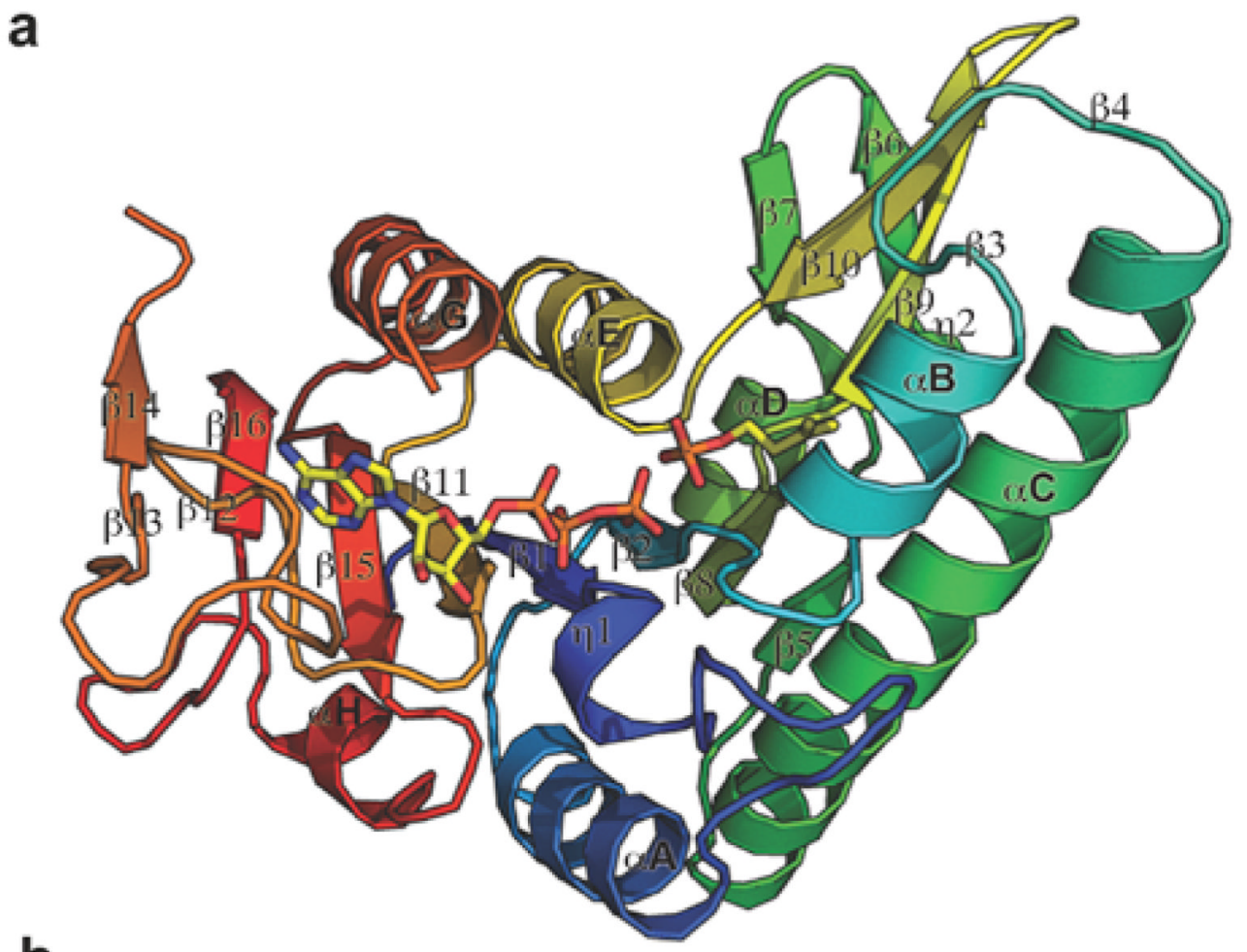

b


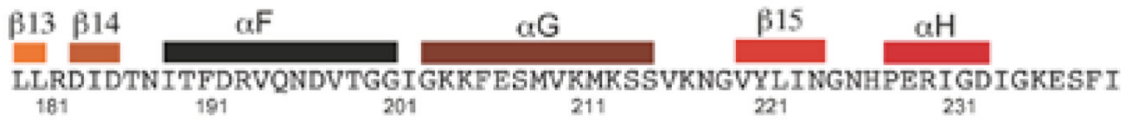

$\frac{\beta 16}{\text { GTVIR }}$

Figure 2.

Tertiary structure of THA IPK. (a) A ribbon diagram of the THA IPK monomer with the helices and strands labeled. The region that connects $\beta 14$ and $\alpha \mathrm{G}$ is disordered in the crystal and not modeled in the structure. (b) The THA IPK primary sequence and secondary structures in the same color scheme as in (a). The residues are numbered below the primary sequence. The segment between $\beta 14$ and $\alpha \mathrm{G}$ (which contains $\alpha \mathrm{F}$ ) is not modeled. 
a



b

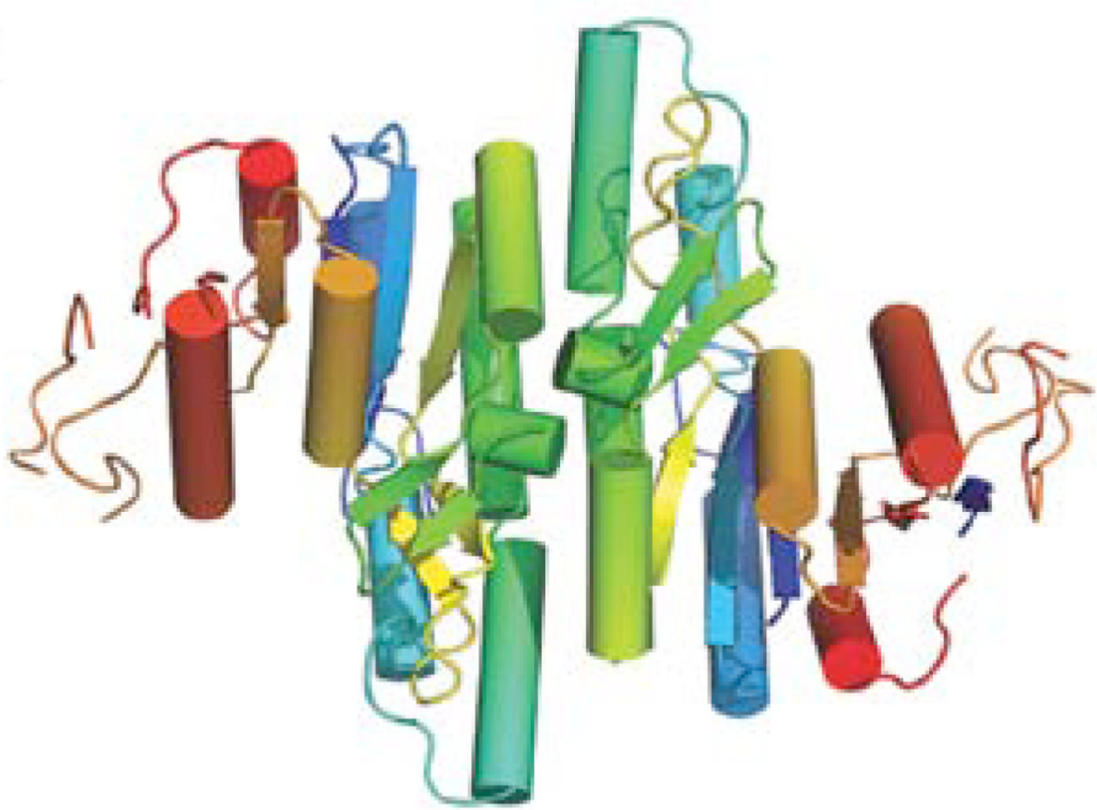

Figure 3.

Homodimers of THA and MTH IPK's. (a) The THA IPK homodimer, with secondary structures involved in the dimer interface labeled. (b) The MTH IPK homodimer. The dyadic axis is perpendicular to the core $\beta$-sheet that runs across the dimer and projects toward the viewer. 


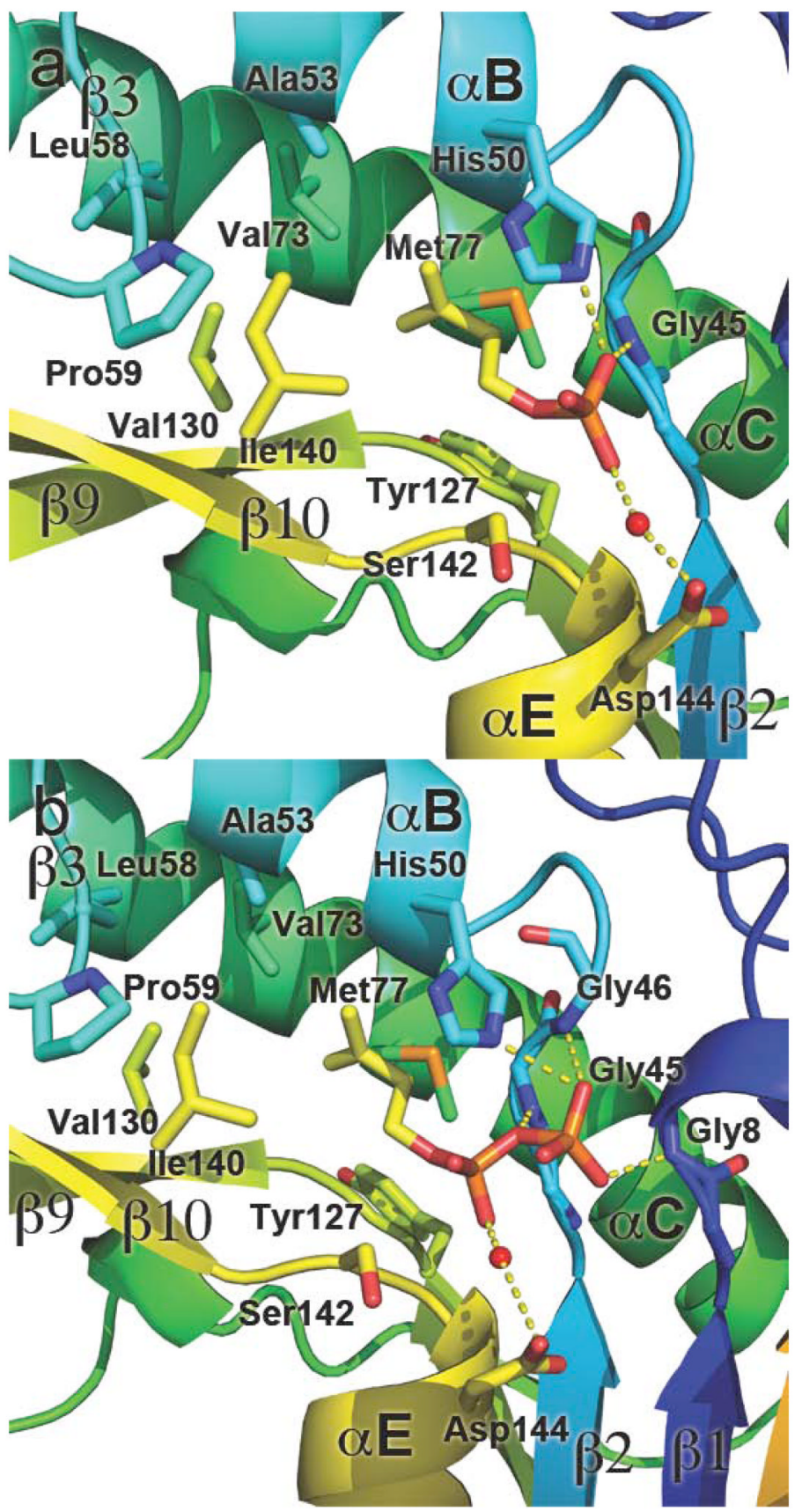

Figure 4.

IP and IPP binding sites (a) The IP binding site showing the amino acid residues that form the hydrophobic interior of the pocket and limit the chain length specificity of the enzyme.

Hydrogen bonds between active site residues and the phosphate moiety of IP are shown as broken yellow lines. A water molecule in the active site is modeled as a red sphere, acting as a hydrogen bonding bridge between IP and Asp144. (b) The IPP binding site. 

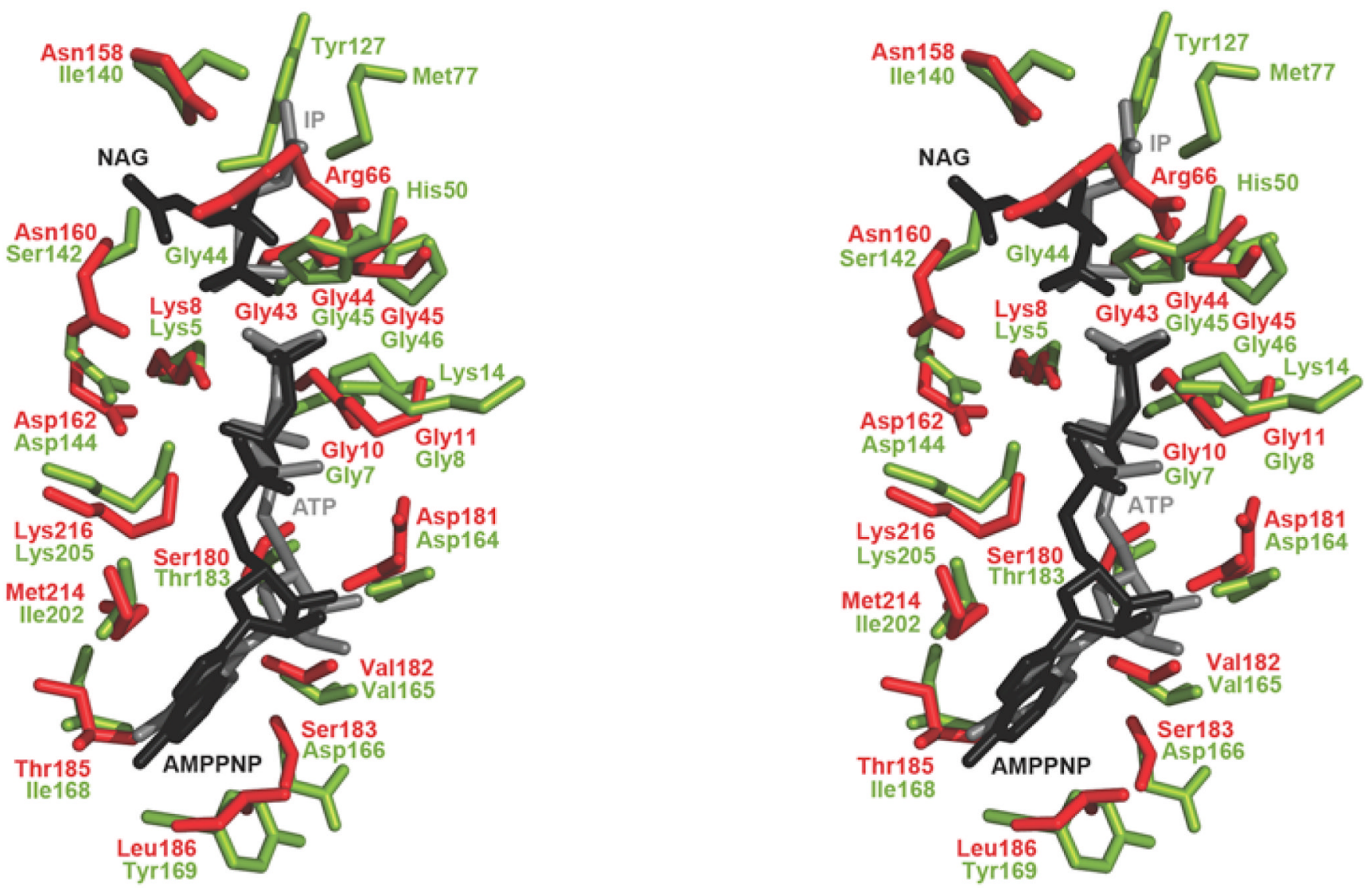

Figure 5.

Stereo view comparisons of THA IPK and NAGK active sites. THA IPK (green) and E. coli NAGK (red) align $207 \mathrm{C} \alpha$ with an r.m.s.d. of $3.0 \AA$ A. Sequence identity is $14 \%$. The bound molecules of THA IPK (IP and ATP) and NAGK (NAG and AMPPNP) align well in the active site. Overlapping active site residues between THA IPK and NAGK are also labeled. Some residues in the IP and NAG binding sites are also modeled, showing residues that give rise to the specificity of these two enzymes. 


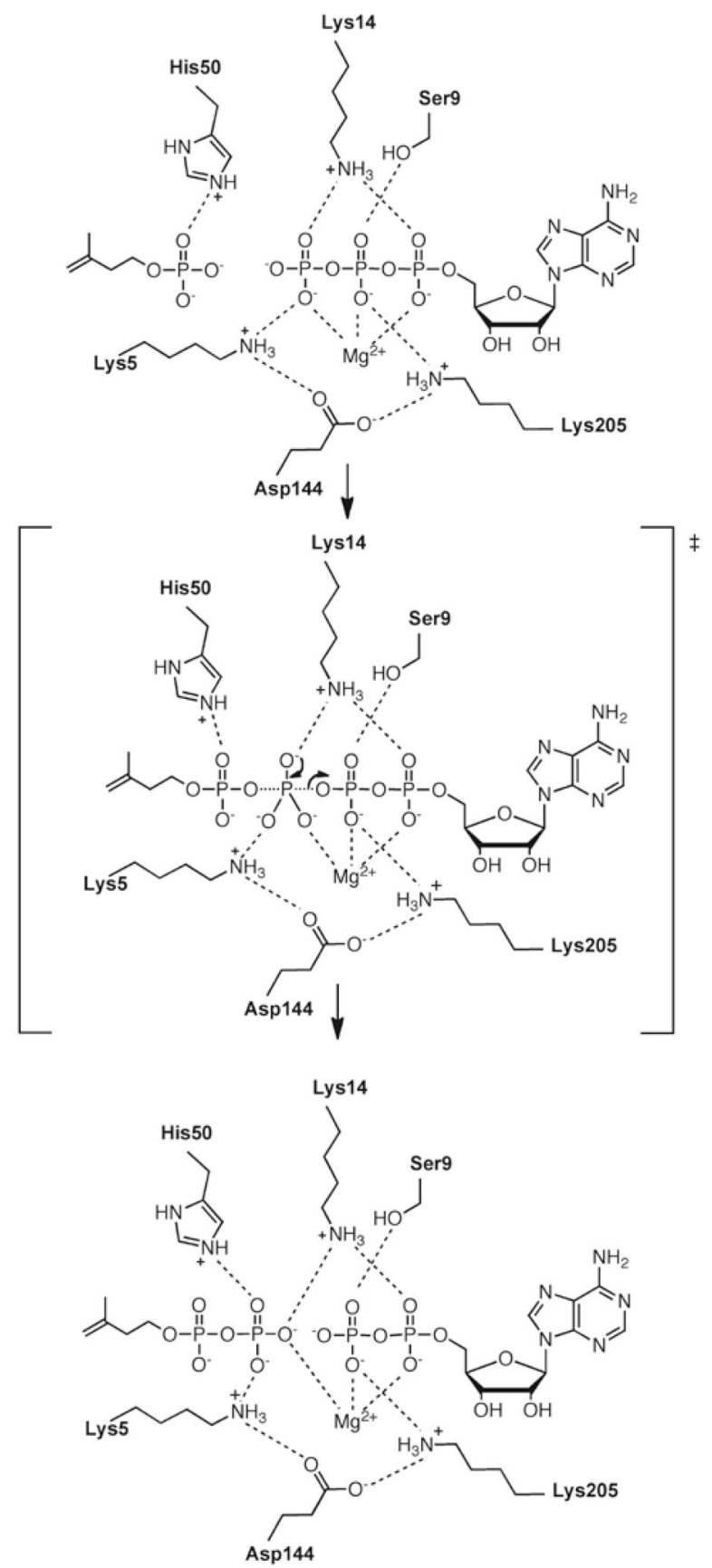

Figure 6.

Proposed catalytic mechanism for IPK. Catalytic residues and selected hydrogen bonds are shown. The "missing" $\mathrm{Mg}^{2+}$ ion is positioned based on overlap with the structure of FomA. 


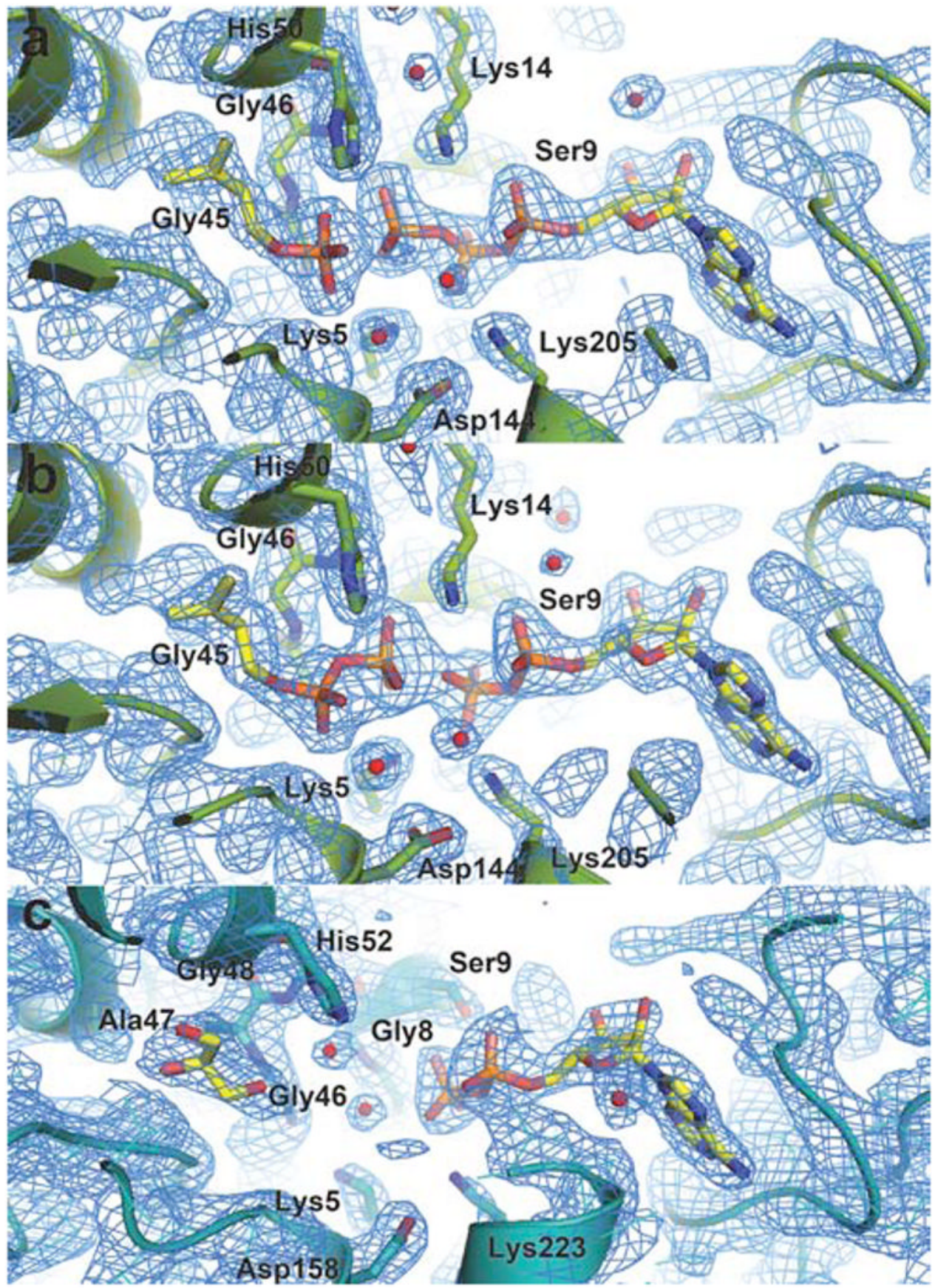

Figure 7.

Electron density maps of the active sites of THA and MTH IPK's contoured at 1 RMSD. (a) The THA IPK active site with bound substrates, IP and ATP. (b) The THA IPK active site with bound products, IPP and ADP. The Gly8 residue is obscured by P $_{\gamma}$ of ATP. (c) The MTH IPK active site with bound glycerol and ADP, showing the putative catalytic residues, Gly8 and Ser9. Putative catalytic residues are labeled and water molecules are shown as red spheres. Ligand omit maps for IP and ATP, and IPP and ADP, can be found in Supplementary Figure 2 , panels $a$ and $b$. 


\section{Table 1}

Data collection and refinement statistics ${ }^{a}$

\begin{tabular}{|c|c|c|c|}
\hline & IPK•IP•ATP & IPK•IPP•ADP & $\mathrm{IPK} \cdot \mathrm{GOL} \cdot \mathrm{ADP}$ \\
\hline \multicolumn{4}{|c|}{ Data Collection } \\
\hline Space group & $\mathrm{C} 2$ & $\mathrm{C} 2$ & $\mathrm{C} 2$ \\
\hline \multirow[t]{4}{*}{ Cell dimensions } & $\mathrm{a}=124.31$ & $\mathrm{a}=187.10$ & $\mathrm{a}=119.67$, \\
\hline & $\mathrm{b}=44.28$ & $\mathrm{~b}=42.79$ & $\mathrm{~b}=96.36$ \\
\hline & $c=91.76$ & $\mathrm{c}=134.90$ & $\mathrm{c}=72.43$ \\
\hline & $\beta=109.45$ & $\beta=113.19$ & $\beta=120.59$ \\
\hline Molecules/asu & 2 & 4 & 2 \\
\hline Wavelength ( $\mathrm{A})$ & 1.54 & 0.97887 & 1.54 \\
\hline Resolution range ( $($ ) & $29.30-2.00$ & $50.00-1.99$ & $30-2.15$ \\
\hline (outer shell) & $(2.07-2.00)$ & (2.06-1.99) & $(2.18-2.15)$ \\
\hline Unique reflections & 31,495 & 65,827 & 38,177 \\
\hline $\mathrm{R}_{\mathrm{sym}} b(\%)$ & $7.5(47.5)$ & $6.20(23.4)$ & $9.1(45.4)$ \\
\hline \multirow{2}{*}{ Completeness $(\%)$} & $98.0(95.2)$ & $94.6(80.9)$ & $92.3(66.2)$ \\
\hline & \multicolumn{2}{|c|}{ Refinement } & \\
\hline Resolution range & $29.30-2.00$ & $38.29-1.99$ & $70-2.15$ \\
\hline $\mathrm{R}_{\text {factor }}^{c}($ work/test $)(\%)$ & $17.5 / 23.1$ & $18.9 / 23.7$ & $20.7 / 26.0$ \\
\hline RMSD bonds $(\AA)$ & 0.022 & 0.017 & 0.022 \\
\hline \multirow[t]{2}{*}{ RMSD angles $\left({ }^{\circ}\right)$} & 1.9 & 1.8 & 2.0 \\
\hline & \multicolumn{3}{|c|}{ Ramachandran plot ${ }^{d}$} \\
\hline Most favored (\%) & 98.70 & 97.81 & 94.02 \\
\hline Allowed (\%) & 1.30 & 2.19 & 5.92 \\
\hline Disallowed (\%) & 0.00 & 0.00 & 0.06 \\
\hline \multicolumn{3}{|c|}{${ }^{a}$ Statistics for highest resolution shell are in parentheses. } & \\
\hline
\end{tabular}

